\title{
Les membres genevois de la «Société médicale d'observation » de Paris (1832)
}

\author{
Par Erwin H. Ackerknecht
}

La «Société médicale d'observation» de Paris, fondée en 1832, est bien connue comme l'organisation qui pendant des décades a propagé avec grand succès la célèbre «méthode numérique» de Pierre-Charles-Alexandre Louis (1787-1872), médecin de la Pitié de 1828-1859. Il est également bien connu que cette méthode, appliquée par Louis surtout dans ses deux chef-d'œuvres sur la phthisie (1825) et sur la fièvre typhoïde (1829), a profondément influencé la médecine clinique jusqu'à nos jours ${ }^{1}$.

Il est beaucoup moins connu que cette organisation de l'avant-garde de la méthode numérique s'est formée sur l'initiative de trois étudiants genevois de Louis : Jacob-Marc d'Espine, Théodore Maunoir et John Bizot ${ }^{2}$. Ensemble avec eux, trois autres étudiants genevois (Baumgartner, J. J. Chaponnière, A. Sestier) devinrent membres fondateurs de la Société. Donc six sur seize membres fondateurs de cette Société Parisienne étaient des Genevois! Bientôt quatre autres Genevois (F. Rilliet, V. Gautier, L. Piachaud, Th. Herpin) joignirent les fondateurs et H.C.Lombard, quoique son appartenance soit incertaine, fut de toute façon un des partisans les plus fidèles de Louis. Quoique la Société en croissant (146 membres en 1856) devint plus «française», l'élément genevois (et anglo-americain !) resta toujours considérable ${ }^{3}$.

Il n'est pas surprenant que le premier volume des Mémoires de la Société est composé de quatre essais dont les auteurs étaient Louis, d'Espine, Bizot et Th. Maunoir. Des onze membres genevois des débuts, six au moins ont fait une contribution scientifique dont l'importance dépasse largement Genève et la Suisse. Avant d'étudier ces six "grands», donnons une courte description des cinq autres.

Antoine Baumgartner (1808-1895)4, Dr. en méd. Paris 1833, grand original, s'est surtout occupé de politique et de questions médicales touchant à la politique comme les logements d'ouvriers, les asiles de vieillards etc.

Jean-Jacques Chaponnière (1805-1859) ${ }^{5}$, Dr. en méd. Paris 1831, s'intéressa surtout à l'histoire de médecine.

Ami-Daniel-Félix Sestier $(1803-1857)^{6}$, Dr. en méd. Paris 1832, resta à Paris et suivit une carrière honorable (Interne, Chef de Clinique, professeur agrégé). Ses livres sur la pneumonie (1840) et l'angine laryngée œéemateuse (1852) sont des contributions respectables dans l'esprit de Louis; son grand livre posthume «De la foudre» (1866) est une œuvre éminente. 
Horace-Charles-Victor Gautier (1824-1890) ${ }^{7}$, Interne des Hôpitaux 1845, Dr. en méd. Paris 1850, fut un chirurgien et obstétricien fort capable. Il avait aussi étudié à Zurich 1841-1843. Ses quatres règles de l'opérateur (1867) écrites sous l'influence de Semmelweis et présageant déjà l'asepsie passèrent malheureusement plus ou moins inaperçues.

Louis Piachaud (1824-1890)8, Interne 1847, Dr. en méd. Paris 1852, fut chirurgien en chef de l'Hôpital cantonal 1866-1870.

Des trois fondateurs de la Société c'est Jacob-Marc d'Espine (1806-1860 ${ }^{9}$ qui a sans doute influencé le plus fortement la science médicale. Interne de Paris en 1831, il publia en 1833 une thèse «Comment un médecin doit-il penser ? Comment doit-il agir ?» qui est une défense théorique de la méthode de Louis plus détaillée que le maître ne l'a jamais donné. Il avait d'ailleurs connu déjà Louis à Odessa, où il avait passé son enfance. Il rassembla aussi à Paris des matériaux cliniques dans le sens de son groupement (voir ses travaux comme celui sur l'orchite blennorrhagique dans le premier volume des Mémoires : ceux sur la leucorrhée, le col de l'utérus, la puberté dans les Archives Générales de 1836). Mais après sa rentrée à Genève en 1834 il lui est impossible de continuer ces recherches en l'absence des grands hôpitaux de Paris, ce qui est d'ailleurs arrivé à tous les disciples genevois et américains de Louis qui ont quitté Paris ${ }^{10}$. Le conseil de santé le chargeant de lui présenter le rapport sur la mortalité du canton de Genève pour l'année 1838 (publié dans les Annales d'Hygiène Publique en 1840), il trouve un nouveau chemin d'application de la méthode numérique : la statistique médicale, surtout mortuaire. Il examine la mortalité selon le lieu d'habitation, les sexes, les saisons, les âges, selon les accidents et les maladies. Il compare, bien sûr, ses résultats avec ceux de Louis concernant la typhoïde et la tuberculose. Il peut se baser sur les 1405 diagnostics donnés par les médecins genevois dans le «livre de l'hôpital» et le «livre de la faculté». La vie moyenne des hommes en 1838 est 35,5 ans, celle des femmes 39,1 ans. Déjà le problème de la classification nosologique se pose. D'Espine continue ces revues annuelles en 1842, 1843, 1844 et 1845. Au Congrès international de statistique de Bruxelles de 1853, sa réputation est déjà telle qu'il est chargé avec le fameux Farr de Londres de soumettre une classification nosologique internationale au Congrès de Paris de 1855. Mais les deux spécialistes n'arrivent pas à se mettre d'accord. En 1857 d'Espine est déjà trop malade pour participer au Congrès de Vienna. Il réussit encore à publier en 1858 son chef-d'œuvre (dédié à Louis) «Essai analytique et critique de statistique mortuaire comparée renfermant les monographies étiologiques des accidents de la plupart des maladies mortelles et expliquant les lois générales de la mortalité 
des peuples, par les influences combinées des diverses causes de mort». C'est tragique que le grand changement dans les notions d'étiologie amené par les découvertes bactériologiques, faites entre 1876 et 1895, ont fait vieillir prématurément ce travail monumental (comme d'ailleurs aussi celui d'August Hirsch).

D'Espine observa en 1833 déjà la différence entre le typhus et la fièvre typhoïde, mais comme il ne publia son observation qu'en 1853 (Recueil des travaux de la Société médicale de Genève 1, p. 119-141) c'est deux autres disciples de Louis, les Américains W.W.Gerhard et C.W.Pennock, publiants en 1837, qui ont fait officiellement cette découverte importante. D'Espine faisait beaucoup pour propager les statistiques médicales en Suisse. Le premier professeur d'hygiène de l'Université de Genève, Pierre-Louis Dunant (1834-1918, un frère d'Henri), était son disciple. Il s'accorde bien avec l'attitude générale de d'Espine que de 1837 à 1856 il était médecin des prisons. Dans sa pratique quotidienne il s'est surtout voué à l'otologie. Il est mort prématurément d'une maladie d'estomac en 1860 .

Le deuxième des trois mousquetaires, John Bizot (1804-1885) ${ }^{11}$ pratiqua après sa promotion à Paris en 1835, la chirurgie et l'obstétrique à Genève. Chirurgien d'hôpital de 1853 à 1859, il s'est (comme d'autres médecins genevois «aristocrates») retiré assez tôt à la campagne. Ce chirurgien n'a publié qu'un seul travail d'importance, sa thèse: «Recherches sur le cœur et le système artériel chez l'homme» (publié aussi dans le vol. I des Mémoires de la Société). Mais ce travail est d'une telle qualité qu'il lui a assuré une place dans les annales de la science. Cette contribution à l'anatomie et l'anatomie pathologique des artères a été discutée par exemple par Virchow, Rokitansky, Hasse, Lebert, Donders et Jansen ${ }^{12}$. Dans cette «enorme Fleißarbeit», comme Müllener l'appelle à juste titre ${ }^{13}$, Bizot observa et surtout mesura dans 157 autopsies une énorme quantité de détails (il mesura par exemple la forme dans 2162 vaisseaux) et apporta un grand nombre de nouveaux faits concernant l'artériosclérose. La thèse de Bizot est une des grandes réussites de la méthode numérique.

Théodore-David-Eugène Maunoir (1806-1869) ${ }^{14}$, le troisième fondateur de la Société, appelé aussi «Maunoir neveu», fut comme son père Charles-Théophile et son oncle Jean-Pierre, un chirurgien et ophthalmologiste réputé. Il finit ses études à Paris en 1833 avec une dissertation: «Essai sur quelques points de l'histoire de la cataracte» (aussi reproduite dans le vol. I des Mémoires), travail solide dans l'esprit de l'école de Louis. Il était chirurgien de l'Hôpital de 1840 à 1843. Il est important surtout par le fait que avec Henri Dunant, Gaston Moynier, le Général Dufour et le Dr. Appia il était membre du premier comité international de la Croix Rouge de 1863 jusqu'à sa mort. 
Théodore-Joseph-Dieudonné Herpin (1799-1865) ${ }^{15}$ étudia à Paris de 1816 à 1823, et y apprit l'auscultation chez Laënnec. Il devint un des fondateurs de la Société médicale de Genève. Il était un praticien très fréquenté et a beaucoup publié. Son intérêt principal était l'épilepsie à laquelle il a voué de nombreux articles pleins de découvertes et deux livres magistraux : «Du pronostic et du traitement curatif de l'épilepsie» (Paris 1852) et «Des accès incomplets d'épilepsie» (Paris 1867). Pour le premier, il reçut un prix de l'Académie des Sciences. Il adopta tôt la méthode de Louis. A vrai dire le mot d'ordre de son livre de 1852 «Exactitude et perséverance» en est peut-être la meilleure définition raccourcie. Il propaga aussi des idées intéressantes d'ordre social comme les visites médicales des communes rurales ou «l'abonnement médical». Il était l'ami de Tœpffer. En 1856, à l'age de 57 ans il transféra son domicile à Paris où il est mort en 1865. Est-ce que c'est un hasard que c'est l'année de «l'épuration» dont nous parlerons plus tard?

Louis-Frédéric-Théodore-Albert Rilliet (1814-1861) ${ }^{16}$ passa de 1833 à 1843 à Paris des années pleines de succès. Il obtint la Médaille d'or de l'internat, le Prix Montyon et le Prix Itard. Sa thèse de 1840 s'occupa de la fièvre typhoïde des enfants, considérée comme rare jusqu'à son travail. En 1843 il publia avec E. Barthez, son co-interne à l'Hôpital des Enfants, le «Traité clinique et pratique des maladies des enfants » qui pendant soixante ans était internationalement reconnu comme le meilleur texte dans la jeune discipline de pédiatrie. Rilliet a donc au delà des contributions de détail précieuses comme la fièvre typhoïde infantile, la méningite tuberculeuse, les deux pneumonies infantiles, et les prodromes des maladies infectieuses bien mérité de la pédiatrie. Rilliet, tout en commençant à douter la position centrale de l'anatomie pathologique, resta fidèle à la méthode de Louis, dont il avait été l'externe. En dépit de ses succès Rilliet rentra à Genève où il devenait un praticien très recherché. De 1848 jusqu'à la «purge» de 1856 il était le médecin de l'Hôpital. Il est malheureusement mort d'une crise cardiaque subite en 1861 n'ayant atteint que l'âge de 41 ans.

Henri-Clermont Lombard (1803-1895) ${ }^{17}$ est avec Marc d'Espine et François Rilliet sans doute le plus éminent des disciples genevois de Louis. Il commença ses études à Edinburgh, mais dut se rendre en Italie, à cause d'une affection pulmonaire, une expérience qui éveilla tôt son intérêt dans la climatologie médicale. Il continua ses études à Paris, finissant en 1827 avec une thèse sur les tubercules sous Andral. Son grand traité de 1878 est dédié à ses deux maîtres Andral et Louis, qui étaient d'ailleurs étroitement liés. Sous l'influence d'Andral il appartenait aussi avec Littré à une société s'adonnant à l'étude de vieux textes médicaux. Obtenant le Prix Montyon en 1829 il rentra à Genève la même année. Il 
était médecin de l'Hôpital de 1835 à 1848. Il a laissé des rapports intéressants sur des épidémies de grippe ou de choléra (il était contagionniste) à l'Hôpital. Mais le nombre des cas était trop petit pour pratiquer avec succès la méthode numérique et Lombard se tourna vers l'analyse quantitative de la climatologie, domaine dans lequel il a beaucoup publié, surtout sur «les climats de montagne considérés au point de vue médical» (Genève 1856). En 1877, à l'âge de 74 ans, il publia son traité monumental de «Climatologie médicale comprenant la météorologie médicale et l'étude des influences physiologiques, pathologiques, prophylactiques et thérapeutiques du climat sur la santé» (Paris 1877-1880, 4 volumes et 1 atlas). Comme dans le cas de Marc d'Espine et d'August Hirsch, les découvertes bactériologiques des années suivantes faisaient malheureusement dater certaines parties du Traité plutôt rapidement. Lombard resta actif jusqu'à un âge très avancé. Il présida le Congrès International d'Hygiène, qui se tint à Genève en 1882. Une contribution importante de Lombard a été malheureusement bien négligée: sa différenciation du typhus et de la fièvre typhoïde («typhus anglais» et «typhus français» comme il les appelait ${ }^{18}$ ) en 1836. Sa contribution n'est pas moindre que celle de W.W.Gerhard, qui faisait la même observation avec Pennock en 1836 et ne la publia qu'en $1837^{19}$.

Que les disciples de Louis étaient et restaient amicalement liés à Genève, est illustré par un dessin spirituel de Rilliet de 1850, donc quinze à vingt ans après leur retour de Paris, dessin qui a été publié par Jean Olivier en $1948^{20}$. Nous y voyons rassemblés autour de la table de Rilliet: Marc d'Espine, Th. Maunoir, J. Bizot, H. C. Lombard, J. J. Chaponnière, Th. Herpin, donc sept des dix «Louisiens» discutés ci-dessus (ensemble avec Chossat, Marcet, Ch. Coindet, André Gosse et J. B. Strœhlin).

Dix ans plus tard quatre des douze convives étaient morts, quatre chassés de leurs postes. Cela s'est fait par «l'épuration» notoire entreprise par le gouvernement de James Fazy en $1856^{21}$ où tous les médecins «aristocrates », même les plus capables, furent privés de leurs postes publics. On procéda de la manière suivante. Le Conseil d'État destitua le 16 mars 1856 le Dr. Ch. Coindet de sa place de Médecin en chef de la maison d'aliénés, qu'il avait honorablement remplie pendant 22 ans, sous l'accusation fausse de «détention arbitraire d'une jeune fille mineure». Le 8 avril 1856 la Faculté de Médecine (l'ensemble des médecins genevois) adressa une lettre de sympathie à Coindet, signée par 55 membres. Seuls n'ont pas signé les sept docteurs appartenant au parti de Fazy, artisans ou profiteurs du coup. Pour avoir signé cette lettre fut déstitué le 9 mai le Dr. Figiuère comme médecin de la gendarmerie, le 29 juin furent exclus le Dr. Rilliet comme médecin, 
le Dr. Strœhlin comme chirurgien de l'Hôpital cantonal, le $1^{\text {er }}$ août on revoqua le Dr. d'Espine comme médecin des prisons.

C'est ainsi que finit une période presque centenaire de l'histoire médicale de Genève, allant de Louis Odier et Louis Jurine à François Rilliet et Marc d'Espine, période pendant laquelle Genève était incontestablement le centre médical le plus développé de toute la Suisse. Quand la nouvelle Faculté ouvrait ses portes le 26 octobre 1876, aucun de ses membres faisait parti de ce passé ; c'était une faculté brillante, mais orientée très différemment et qui ne jouit plus de la situation prépondérante de ses prédécesseurs.

Quelquefois on se demande pourquoi, sauf Sestier, aucun de ces disciples de Louis après des débuts brilliants n'est resté à Paris. Ils sont tous rentrés à Genève. Nous ignorons leurs raisons, vraisemblablement souvent personnelles, quelquefois peut-être religieuses. Mais il nous semble que les difficultés qu'ils rencontraient à Genève leur suggéraient peut-être des initiatives plus originales que les routines parisiennes n'auraient faites.

\section{Notes}

${ }^{1}$ Ackerknecht, Erwin H., Medicine at the Paris Hospital 1794-1848, Baltimore 1967, p. 102-104.

Astruc, Pierre, P.Ch.A.Louis, in: Les biographies médicales, Paris 1933, $\mathrm{N}^{\circ} 7$.

Delaunay, Paul, Louis et la méthode numérique, in: Science, Medicine and History, London 1953, p. 321-330.

${ }^{2}$ Louis, Pierre, Avertissement, in : Mémoires de la Société Médicale d'Observation, vol. I, Paris 1837, p. IX.

Je me suis occupé à différentes reprises des disciples genevois de Louis :

Ackerknecht, Erwin H., The Paris Clinical School, in : Year Book Am. Phil. Soc. for 1951, Philadelphia 1952, p. $257 \mathrm{ff}$.

id., Die Pariser Spitäler als Ausgangspunkt einer neuen Medizin, in: Ciba Sympos. 7 (1959), p. 98-105.

id., La médecine à Genève dans la première moitié du XIX ${ }^{\mathrm{e}}$ siècle, in: XIX ${ }^{e}$ Congr. int. Histoire de la méd., Basel 1964, p. 420-425, Basel/New York 1966.

id., Grands Médecins Suisses 1500-1900. Confér. Palais de la Découverte D 109, Paris 1966. Ackerknecht, Erwin H., und Buess, Heinrich, Kurze Geschichte der großen Schweizer Ärzte, Bern 1975, p. 46-55.

Quand le Dr. E.R.Müllener de Berne était mon assistant en 1962-1963, j'ai attiré son attention sur quelques aspects de l'histoire de médecine de Genève : le psychiatre Matthey, les travaux genevois sur la meningite, les disciples genevois de Louis et certaines simi- 
larités avec les disciples américains de Louis. Le Dr. Müllener a publié au cours des années soixante plusieurs articles sur ces sujets, dont la liste suit. Je lui dois la connaissance d'un nombre de faits inconnus avant ses recherches consciencieuses.

Müllener, Eduard-Rudolf, Die Entstehung des Kleptomaniebegriffs, in : Sudhoffs Archiv 48 (1964), p. 216-239.

id., Six Geneva physicians on meningitis, in : J. Hist. Med. 20 (1965), p. 1-26.

id., Genfer Medizinalstatistik und Hygiene in der ersten Hälfte des 19. Jahrhunderts, in : Gesnerus 21 (1964), p. 154-192.

id., P.-Ch.-A.Louis' Genfer Schüler und die «méthode numérique», in : Gesnerus 24 (1967), p. 46-74.

Autres publications qui s'occupent partiellement des disciples genevois de Louis:

Martino, E., Olivier, J., Roch, M., $125^{e}$ anniversaire de la Soc. Méd. de Genève, Genève 1948.

Olivier, J., Aperçu de l'histoire de la médecine à Genève. Cours de Perfect. Fac. méd. Genève 1943, p. 711-724.

Picot, C., et Thomas, E., Centenaire de la Soc. Méd. de Genève 1823-1923, Genève 1923.

Thomas, E., La médecine à Genève au début du XIX ${ }^{e}$ siècle, Basel 1937.

${ }^{3}$ Mémoires de la Soc. Méd. d'Observ., vol. III, Paris 1856.

${ }^{4}$ Baumgartner, A. J., Le Dr. Baumgartner, Genève 1895.

${ }^{5}$ Picot et Thomas l.c., p. 143 ; Müllener l.c., 1966, p. 62.

${ }^{6}$ Müllener 1.c., 1966, p. 63-65.

${ }^{7}$ Picot, C., Le Dr. V. Gautier, in : Revue méd. Suisse Romande 10 (1890), p. 122-130.

${ }^{8}$ Picot et Thomas 1.c., p. 111-112.

${ }^{9}$ Dupin, J.P., Le Dr. Marc d'Espine. Notice biographique, publié par la Soc. Méd. du Canton de Genève, 1860.

${ }^{10}$ Ackerknecht, Erwin H., Elisha Bartlett and the philosophy of the Paris Clinical School, in: Bull. Hist. Med. 24 (1950), p. 43-60.

${ }^{11}$ Nécrologie du Dr. J. Bizot, in : Revue méd. Suisse Romande 5 (1885), p. 616.

12 Hofer, Hans-Ulrich, Die Arteriosklerose in der pathologischen Anatomie des 19. Jahrhunderts. Zürcher Med. gesch. Abh., NS Nr. 99, 1974, p. 28.

${ }^{13}$ Müllener l.c., 1966, p. 61.

${ }^{14}$ Duval, A.J., Notice sur le Dr. Th.Maunoir, Lausanne 1869.

${ }^{15}$ Dufresne, E., Th. Herpin, in : Comptes rendus trav. Soc. Méd. Genève, 1865, p. 28-33.

${ }^{16}$ Duval, J., Le docteur Rilliet, Genève 1861.

Fischer, Pius, Der «Traité clinique des maladies des enfants» von Rilliet und Barthez.

Zürcher Med. gesch. Abh., NS Nr. 37, 1960.

${ }^{17}$ Picot, C., Le Dr. Henri-Clermont Lombard, in : Revue méd. Suisse Romande 15 (1895), p. 116-123. 
${ }^{18}$ Lombard, Henri Clermont, Observations on Typhous Fever, Dublin 1836.

19 Am. J. Med. Sc. 19 (1837), p. 302.

20 in Martin, Olivier, Roch l.c., 1948, p. 36-39.

${ }^{21}$ Société Médicale de Genève. Documents sur la position faite par l'administration publique à la Faculté de médecine de Genève. Genève 1856.

\section{Summary}

The famous "Société médicale d'observation" at Paris was founded by three Geneva pupils of P.Ch.A.Louis, the creator of "la méthode numérique". Eight more Geneva students were among his most faithful followers. Life and work of the six most eminent men of this group of eleven : M. d'Espine, J. Bizot, Th. Maunoir, Th. Herpin, F. Rilliet and H. C. Lombard are reported in some detail. They represent the last peak of that great one hundred year phase of Geneva medicine going from Odier and Jurine to Rilliet and d'Espine. Rilliet and d'Espine lost their hospital positions in the political "purge" of 1856.

Prof. Dr. Erwin H. Ackerknecht

Ottikerstraße 42

8006 Zürich 